\title{
COVID-19 and the endocrine system: exploring the unexplored
}

\author{
R. Pal ${ }^{1} \cdot$ M. Banerjee ${ }^{2}$ \\ Received: 8 April 2020 / Accepted: 23 April 2020 / Published online: 2 May 2020 \\ (C) Italian Society of Endocrinology (SIE) 2020
}

Keywords COVID-19 · Endocrinology · Adrenal $\cdot$ Thyroid diseases $\cdot$ Pituitary diseases · Diabetes mellitus

\section{Introduction}

A novel coronavirus disease (COVID-19), caused by severe acute respiratory syndrome coronavirus 2 (SARS-CoV-2), has affected over 2.3 million people, claiming more than 160000 lives in over 200 countries worldwide. Although COVID-19 can be asymptomatic, the primary presentation in symptomatic patients is in the form of viral pneumonia, sometimes complicated by acute respiratory distress syndrome and/or sepsis. In addition, myocarditis and acute kidney injury have also been described in COVID-19. On the contrary, endocrinological manifestations have not yet been reported in patients with COVID-19.

The pathogenesis of COVID-19 entails entry of SARSCoV-2 via the respiratory system and lodgement in the lung parenchyma. Thereafter, it uses angiotensin-converting enzyme 2 (ACE2) as a receptor for ingress into host pneumocytes. In addition, the viral ribonucleic acid (RNA) has also been detected in the plasma or serum of COVID-19 patients, suggestive of viremia [1]. This implies that apart from pneumocytes, the virus is freely available to interact with ACE2 expressed in other tissues. As a matter of fact, a number of endocrine organs do express ACE2, namely pancreas, thyroid, testis, ovary, adrenal glands and pituitary [2]. Even though one could expect endocrine repercussions due to interaction of SARS-CoV-2 with ACE2 expressed on these organs, there are no clinical/pre-clinical data as of yet. However, based on observations drawn partly from studies

R. Pal

rimesh.ben@gmail.com

1 Department of Endocrinology, Post Graduate Institute of Medical Education and Research (PGIMER), Chandigarh 160012, India

2 Department of Endocrinology, Institute of Post Graduate Medical Education and Research (IPGMER), Kolkata 700007, India pertaining to the prior SARS outbreak (in 2003) and partly from animal models, we aim to explore the possible effects of COVID-19 on the endocrine system.

\section{COVID-19 and endocrine pancreas}

ACE2 is expressed in pancreas with mRNA levels being higher in pancreas than in the lungs. The expression is seen on the exocrine pancreas as well as on the islets. Exocrine pancreatic injury is manifested as elevated serum amylase and/or lipase in 1-2\% and $17 \%$ of patients with non-severe and severe COVID-19, respectively [2]. Although any major illness can be associated with stress-related hyperglycemia, Yang et al. had reported that patients with SARS (caused by SARS-CoV, the 'cousin' of SARS-CoV-2) who had never received glucocorticoids had significantly higher fasting plasma glucose levels as compared to patients with non-SARS pneumonia [3]. In another study, SARS-CoVmediated damage of the pancreatic $\beta$-cells was proposed as the plausible mechanism behind the development of 'acute diabetes' in patients with SARS [4]. In fact, immunohistochemistry and in situ hybridization have identified SARS$\mathrm{CoV}$ in the pancreas of patients who died of SARS [5]. Thus, although coronavirus does not feature in the list of viruses implicated in the etiopathogenesis of type 1 diabetes mellitus (T1DM), SARS-CoV (and perhaps SARS-CoV-2) could be potential environmental triggers for the development of T1DM. Apart from direct $\beta$-cell damage, alterations in selfantigens and subsequent immune-mediated destruction of $\beta$-cells could be implicated. In addition, infection of the surrounding exocrine pancreas by SARS-CoV and SARSCoV-2 might cause a bystander $\beta$-cell death via release of mediators such as tumor-necrosis factor- $\alpha(\mathrm{TNF} \alpha)$ and interferon- $\gamma[6]$. A systemic pro-inflammatory milieu, as evident by high amounts of interleukin-1 $\beta$, monocyte chemoattractant protein-1 (MCP-1) and inducible protein-10 even in 
patients with mild COVID-19 might play an additional role to accentuate the process.

COVID-19 could also lead to worsening of insulin resistance in patients with pre-existing type 2 diabetes mellitus (T2DM). Apart from inducing a plethora of cytokines, SARS-CoV increases serum levels of fetuin A, a glycoprotein that has been linked with impaired insulin sensitivity [7]. Lopinavir-ritonavir used for the treatment of COVID19 could lead to lipodystrophy and subsequent insulin resistance. Moreover, COVID-19 is often associated with hypokalemia; this has been attributed to downregulation of pulmonary ACE2, reduced angiotension-II degradation and subsequent increased aldosterone secretion [8]. Hypokalemia, in turn, can worsen glucose control in patients with pre-existing T1DM and T2DM.

The presence of diabetes mellitus in patients with COVID-19 is associated with severe disease, acute respiratory distress syndrome (ARDS) and increased mortality. Possible explanations include compromised innate immunity and downregulated ACE2 levels in people with diabetes mellitus [8]. In addition, serum levels of inflammationrelated biomarkers (interleukin-6, serum ferritin, C-reactive protein) and coagulation parameter (D-dimer) are higher in COVID-19 patients with underlying diabetes mellitus compared with those without, suggesting that people with diabetes are more susceptible to cytokine storm ultimately leading to ARDS and rapid deterioration [9]. Hitherto available studies do not make any distinction between T1DM and T2DM and it is likely that the COVID-19-related risks holds true for both the disease entities [10]. Nevertheless, it is imperative that people with diabetes mellitus (T1DM and T2DM) take extra precautions and stringently implement social distancing and hand hygiene amid this pandemic. In addition, good glycemic control should be ensured. Certain anti-diabetic drugs like pioglitazone (via peroxisome proliferator-activated receptor $\gamma$ activation) and liraglutide (via glucagon-like peptide 1 receptor activation) have been shown to upregulate ACE2 in animal models [11, 12]; however, the current evidence does not support any change in the ongoing medications [13]. Similarly, international organizations recommend patients on ACEi/ARBs to carry on with their medications.

Obesity has also been found to be associated with severe disease in COVID-19. Adipose tissue express ACE2; with higher adipose tissue, more would be the overall ACE2 expression that would act as receptors for SARS-CoV-2 [14]. As in diabetes mellitus, even in basal state, obese patients have a higher concentration of several pro-inflammatory cytokines such as TNF $\alpha$, IL- 6 and MCP-1, produced by visceral and subcutaneous adipose tissue [15]. This could again predispose an obese individual to an exaggerated cytokine response in the presence of SARS-CoV-2, manifesting as severe disease and ARDS. In addition, obesity is associated with subclinical hypothyroidism and functional hypogonadism that, at least in part, is mediated by cytokines [16, 17]. These could be aggravated amid a pro-inflammatory milieu induced by COVID-19.

\section{COVID-19 and gonads}

A high level of ACE2 expression is seen in the testes; in fact, the mRNA and protein expression of ACE2 in the testis is almost the highest in the human body. Moreover, the Leydig cells, Sertoli cells and the spermatogonia all express ACE2. Nevertheless, serum testosterone levels in COVID-19 needs to be interpreted cautiously, as any acute critical illness can lead to suppression of the hypothalamic-pituitary-testicular axis, biochemically manifesting as low luteinizing hormone (LH), follicle-stimulating hormone (FSH) and testosterone. However, a recent study in 81 men with COVID-19 showed that serum total testosterone (T) was lower (although not statistically significant), while serum LH was significantly higher as compared to 100 age-matched healthy men. Serum $\mathrm{T}: \mathrm{LH}$ ratio was also significantly lower in COVID-19 patients and was negatively associated with disease severity [18]. Elevated serum LH in men with COVID-19 negates the possibility of suppression of the hypothalamic-pituitary-testicular axis and hints toward primary Leydig cell damage. In accordance with this observation, it is to be noted that orchitis was indeed a known complication of SARS [19]. In addition, SARS-CoV infection was shown to significantly reduce serum testosterone in male mice [20]. Nevertheless, data on female gonadal function in women with COVID-19 (or SARS) is not available.

\section{COVID-19 and adrenal gland}

One of the primary immunoinvasive strategy utilized by the SARS-CoV, like the influenza virus, is to knock down the host's cortisol stress response. A very interesting hypothesis that had been proposed is the expression of certain amino acid sequences by the SARS-CoV that are molecular mimics of the host adrenocorticotropic hormone (ACTH). This form of molecular mimicry can indeed blunt the stress-induced cortisol rise, as antibodies produced against the viral particles will inadvertently destroy the circulating ACTH [21]. The fact that most of the SARS-CoV-2 proteins are highly homologous $(95-100 \%)$ to the proteins of the original SARS-CoV makes us wonder whether SARS-CoV-2 might be employing the same strategy of molecular mimicry as well [22]. Therefore, patients with severe COVID-19 may be more prone to develop critical illness-related corticosteroid insufficiency (CIRCI). Data on cortisol dynamics in patients with COVID-19 are however not yet available. Nevertheless, 
clinicians must be vigilant about the possibility of an underlying relative cortisol deficiency in patients with COVID19. Notably, indiscriminate use of short-duration, high-dose glucocorticoids during the SARS outbreak was questioned and not found to be universally useful. One recent study in 31 patients with COVID-19 showed that corticosteroid treatment was not associated with virus clearance time, length of hospital stay or duration of symptoms [23]. Another clinical trial on the efficacy and safety of corticosteroids in COVID19 is currently underway (NCT04273321). Nevertheless, patients with underlying primary adrenal insufficiency (PAI) are at a high risk of lower-respiratory tract infections and hence should take extra precautions amid the ongoing pandemic. They should be aware of sick-day guidelines and increase the dose of corticosteroids by themselves whenever suspected of having COVID-19 to avoid an impending adrenal crisis. PAI patients developing COVID-19 may require parenteral glucocorticoid support; serum potassium should be strictly monitored in such patients, as hypokalemia has been reported in patients with COVID-19 [8].

\section{COVID-19 and the hypothalamus-pituitary}

Neurological manifestations do occur in patients with COVID-19 and include, among others, hyposmia. Expression of ACE2 by the olfactory epithelial supporting cells could explain much of these olfactory symptoms [24]. The portal of entry of the virus into the central nervous system (CNS) remains uncertain and could be via hematogenous route or directly thorough the cribriform plate. Hypothalamic and pituitary tissues do express ACE2 and can theoretically be the viral targets. In fact, on autopsy studies, edema and neuronal degeneration along with identification of SARS genome have been shown in the hypothalamus. Biochemical evidence of hypothalamo-pituitary involvement in SARS was first reported by Leow et al. in 2005. Sixty-one survivors of SARS were evaluated at 3 months post-recovery and thereafter periodically. Forty percent of patients had evidence of central hypocortisolism, the majority of which $(62.5 \%)$ resolved within a year. Of note, $87.5 \%$ of those with central hypocortisolism had experienced fatigue and/or postural dizziness at the time of initial recruitment. A small percentage of patients (5\%) also had central hypothyroidism. The authors had proposed the possibility of a reversible hypophysitis or a direct hypothalamic damage that could have led to a state of hypothalamo-pituitary dysfunction [25]. Currently, we do not have any such data with regard to patients with COVID-19; however, considering the high frequency of neurological symptoms, one can assume that SARS-CoV-2 may affect the hypothalamus-pituitary as well, directly or via immune-mediated hypophysitis. Accordingly, clinicians should have a low threshold to suspect central hypocortisolism in COVID-19 survivors, especially those complaining of unexplained fatigue, lassitude, malaise, orthostatic dizziness, anorexia and apathy. Patients with pituitary-hypothalamic disorders often have underlying diabetes insipidus (DI); COVID-19 in patients with DI can lead to insensible water loss due to fever and tachypnea ultimately resulting in hypernatremia [26]. Hence, the patient and the treating physician need to be cautious in this regard.

\section{COVID-19 and thyroid}

Data on thyroid involvement by coronavirus is most scarce. A study conducted during the SARS outbreak in $2003 \mathrm{had}$ reported that serum T3 and T4 levels were lower in patients with SARS as compared to controls both during the acute and convalescent phases. This could simply imply an underlying sick-euthyroid syndrome. Intensive care patients with sick-euthyroid syndrome tend to have lower mean thyroid weight as a result of reduction in thyroid follicular size associated with depletion of colloid [27]. However, an autopsy study in five patients with SARS has shown marked destruction of the follicular and parafollicular cells of thyroid [28]. Destruction of follicular cells would manifest as low T3 and T4; parafollicular cell damage would theoretically lead to low levels of serum calcitonin. This has been proposed as a plausible mechanism of osteonecrosis of femoral head seen in recovered patients with SARS; calcitonin deficiency leads to disinhibition of osteoclasts leading to osteonecrosis [28]. Data on thyroid function or thyroid pathology are yet not available in COVID-19. The British Thyroid Association and the Society for Endocrinology (BTA/SfE) have issued a consensus statement regarding issues specific to thyroid dysfunction during COVID-19 pandemic. Patients with underlying hypothyroidism or hyperthyroidism are advised to continue their prescribed medications as usual. However, patients on anti-thyroid drugs (ATDs) are at a risk of agranulocytosis, albeit rarely. Symptoms of agranulocytosis often overlap with those of COVID-19, hence, often making it difficult to differentiate one from the other clinically. Hence, it is recommended that patients on ATDs who develop symptoms suggestive of agranulocytosis should immediately discontinue the drug and get a full blood count done at the earliest.

\section{Conclusions}

Amid the ongoing pandemic, endocrine involvement with COVID-19 remains largely unexplored. The aforementioned data pertaining to COVID-19 and the endocrine system are mostly conjectural and factual at this point of time. Validated conclusions must not be drawn based on the presented data, 
as much of the observations are based on prior experience with SARS and on recent literature derived from small-scale studies. However, the data do provide ample scope for future research. As premature as it may sound, endocrinologists need to be aware of these possibilities in clinical practice, especially while dealing with COVID-19 survivors.

\section{Acknowledgements None.}

Author contributions RP is the primary author. MB helped in literature search. Both RP and MB approved the final version of the manuscript.

\section{Funding None.}

\section{Compliance with ethical standards}

Conflicts of interest None to declare.

Ethical approval This article does not contain any studies with human participants or animals performed by any of the authors.

Informed consent For this type of study formal consent is not required.

\section{References}

1. Chang L, Yan Y, Wang L (2020) Coronavirus disease 2019: coronaviruses and blood safety. Transfus Med Rev [Internet] [cited 2020 Apr 1]; Available from: https://linkinghub.elsevier. com/retrieve/pii/S0887796320300146. Accessed 1 Apr 2020

2. Liu F, Long X, Zou W, Fang M, Wu W, Li W, et al. (2020) Highly ACE2 expression in pancreas may cause pancreas damage after SARS-CoV-2 infection [Internet] [cited 2020 Apr 1]. Available from: https://medrxiv.org/lookup/ doi/10.1101/2020.02.28.20029181. Accessed 1 Apr 2020

3. Yang JK, Feng Y, Yuan MY, Yuan SY, Fu HJ, Wu BY et al (2006) Plasma glucose levels and diabetes are independent predictors for mortality and morbidity in patients with SARS. Diabet Med 23:623-628

4. Yang J-K, Lin S-S, Ji X-J, Guo L-M (2010) Binding of SARS coronavirus to its receptor damages islets and causes acute diabetes. Acta Diabetol 47:193-199

5. Ding Y, He L, Zhang Q, Huang Z, Che X, Hou J et al (2004) Organ distribution of severe acute respiratory syndrome(SARS) associated coronavirus(SARS-CoV) in SARS patients: implications for pathogenesis and virus transmission pathways. J Pathol 203:622-630

6. Jaeckel E, Manns M, Herrath M (2006) Viruses and diabetes. Ann N Y Acad Sci 958:7-25

7. Wan J, Sun W, Li X, Ying W, Dai J, Kuai X et al (2006) Inflammation inhibitors were remarkably up-regulated in plasma of severe acute respiratory syndrome patients at progressive phase. Proteomics 6:2886-2894

8. Pal R, Bhansali A (2020) COVID-19, diabetes mellitus and ACE2: the conundrum. Diabetes Res Clin Pract 162:108132

9. Guo W, Li M, Dong Y, Zhou H, Zhang Z, Tian C et al (2020) Diabetes is a risk factor for the progression and prognosis of COVID-19. Diabetes Metab Res Rev 7:e3319

10. Coronavirus COVID-19 FAQ I ADA. Accessed April 21, 2020. [Internet]. Available from: https://www.diabetes.org/covid-19faq. Accessed 21 Apr 2020
11. Zhang W, Xu Y-Z, Liu B, Wu R, Yang Y-Y, Xiao X-Q et al (2014) Pioglitazone upregulates angiotensin converting enzyme 2 expression in insulin-sensitive tissues in rats with high-fat diet-induced nonalcoholic steatohepatitis. Sci World J 2014:1-7

12. Romaní-Pérez M, Outeiriño-Iglesias V, Moya CM, Santisteban P, González-Matías LC, Vigo E et al (2015) Activation of the GLP-1 receptor by liraglutide increases ACE2 expression, reversing right ventricle hypertrophy, and improving the production of SP-A and SP-B in the lungs of type 1 diabetes rats. Endocrinology 156:3559-3569

13. Pal R, Bhadada SK (2020) Should anti-diabetic medications be reconsidered amid COVID-19 pandemic? Diabetes Res Clin Pract 163:108146

14. Kassir R (2020) Risk of COVID-19 for patients with obesity. Obes Rev [Internet] [cited 2020 Apr 22]; Available from: https ://doi.wiley.com/10.1111/obr.13034. Accessed 22 Apr 2020

15. Richard C, Wadowski M, Goruk S, Cameron L, Sharma AM, Field CJ (2017) Individuals with obesity and type 2 diabetes have additional immune dysfunction compared with obese individuals who are metabolically healthy. BMJ Open Diabetes Res Care 5:e000379

16. Sanyal D, Raychaudhuri M (2016) Hypothyroidism and obesity: an intriguing link. Indian J Endocrinol Metab 20:554

17. Fernandez CJ, Chacko EC, Pappachan JM (2019) Male obesityrelated secondary hypogonadism-pathophysiology. Clinical Implications and Management. Eur Endocrinol 15:83

18. Ma L, Xie W, Li D, Shi L, Mao Y, Xiong Y, et al. (2020) Effect of SARS-CoV-2 infection upon male gonadal function: a single center-based study [Internet] [cited $2020 \mathrm{Apr}$ 2]. Available from: https://medrxiv.org/lookup/doi/10.1101/2020.03.21.20037267. Accessed 2 Apr 2020

19. Xu J, Qi L, Chi X, Yang J, Wei X, Gong E et al (2006) Orchitis: a complication of severe acute respiratory syndrome (SARS) 1 . Biol Reprod 74:410-416

20. Channappanavar R, Fett C, Mack M, Ten Eyck PP, Meyerholz DK, Perlman S (2017) Sex-based differences in susceptibility to severe acute respiratory syndrome coronavirus infection. $\mathbf{J}$ Immunol 198:4046-4053

21. Wheatland R (2004) Molecular mimicry of ACTH in SARSimplications for corticosteroid treatment and prophylaxis. Med Hypotheses 63:855-862

22. Xu J, Zhao S, Teng T, Abdalla AE, Zhu W, Xie L et al (2020) Systematic comparison of two animal-to-human transmitted human coronaviruses: SARS-CoV-2 and SARS-CoV. Viruses $12: 244$

23. Zha L, Li S, Pan L, Tefsen B, Li Y, French N, et al. (2020) Corticosteroid treatment of patients with coronavirus disease 2019 (COVID-19). Med J Aust [Internet] [cited 2020 Apr 22]; Available from: https://doi.wiley.com/10.5694/mja2.50577. Accessed 22 Apr 2020

24. Brann DH, Tsukahara T, Weinreb C, Logan DW, Datta SR (2020) Non-neural expression of SARS-CoV-2 entry genes in the olfactory epithelium suggests mechanisms underlying anosmia in COVID-19 patients [Internet] [cited 2020 Apr 2]. Available from: https://biorxiv.org/lookup/ doi/10.1101/2020.03.25.009084. Accessed 2 Apr 2020

25. Leow MK-S, Kwek DS-K, Ng AW-K, Ong K-C, Kaw GJ-L, Lee LS-U (2005) Hypocortisolism in survivors of severe acute respiratory syndrome (SARS). Clin Endocrinol (Oxf) 63:197-202

26. Kaiser UB, Mirmira RG, Stewart PM (2020) Our Response to COVID-19 as Endocrinologists and Diabetologists. J Clin Endocrinol Metab [Internet] [cited 2020 Apr 16];105. Available from: https://academic.oup.com/jcem/article/doi/10.1210/cline m/dgaa148/5814115. Accessed 16 Apr 2020 
27. De Jongh F, Jobsis A, Elte $\mathbf{J}$ (2001) Thyroid morphology in lethal non-thyroidal illness: a post-mortem study. Eur J Endocrinol 144:221-226

28. Wei L, Sun S, Xu C, Zhang J, Xu Y, Zhu H et al (2007) Pathology of the thyroid in severe acute respiratory syndrome. Hum Pathol 38:95-102
Publisher's Note Springer Nature remains neutral with regard to jurisdictional claims in published maps and institutional affiliations. 